\title{
SEARCH FOR LINEAR POLARIZATION OF THE COSMIC BACKGROUND RADIATION
}

P. M. Lubin and G. F. Smoot

WEIVED

Awhenhe

October 1978

GHERT LAOORATORY

noy 1978

Prepared for the U., S. Department of Energy

under Contract W-7405-ENG-48

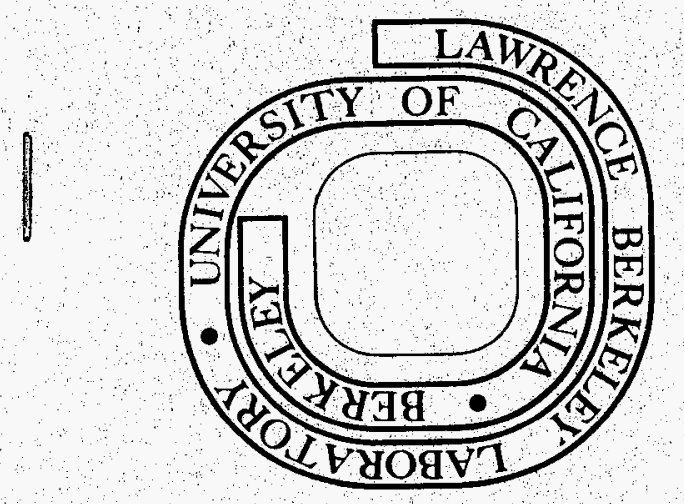

\section{TWO-WEEK LOAN COPY}

This is a Library Círculating Copy which may be borrowed for two weeks. For a personal retention copy, call Tech. Info. Dívision, Ext. 6782 
SEARCH FOR LINEAR POLARIZATION OF THE COSMIC BACKGROUND RADIATION

Phil M. Lubin and George F. Smoot

Space Sciences Laboratory and Lawrence Berkeley Laboratory University of California

Berkeley, California 94720

Received

\section{$\underline{\text { ABSTRACT }}$}

We present preliminary measurements of the linear polarization of the cosmic microwave background $\left(3^{\circ} \mathrm{K}\right.$ blackbody) radiation. These ground-based measurements are made at $9 \mathrm{~mm}$ wavelength. We find no evidence for linear polarization, and set an upper limit for a polarized component of $0.8 \mathrm{~m}^{\circ} \mathrm{K}$ with a $95 \%$ confidence level. This implies that the present rate of expansion of the Universe is isotropic to one part in $10^{6}$, assuming no re-ionization of the primordial plasma after recombination.

The observed cosmic microwave background radiation is generally thought to be a relic of the hot, dense initial phase of the Universe. This radiation should then have characteristics which reflect its thermal origin and the geometry of the early Universe. The simple hot Big Bang model predicts that this radiation has a blackbody (Planckian) spectrum, is unpolarized, exhibits the statistical properties of blackbody radiation ${ }^{1}$, and is isotropic in a reference frame co-moving with the expansion of the universe.

The spectrum of this radiation has been extensively investigated. ${ }^{2}$ The best experiment to date is that of D. Woody and P. Richards. ${ }^{3}$ Although their results qualitatively follow a blackbody spectrum, they report a 50 deviation from the best fit blackbody curve. There have also been many measurements of the angular distribution of this radiation with recent reports of a $3.5 \pm 0.4 \mathrm{~m}^{\circ} \mathrm{K}$ amplitude first-order (cosine) anisotropy ${ }^{4,5}$. It is thought 
that this anisotropy is a result of the Doppler shift caused by the motion of the solar system relative to the cosmic radiation. If this interpretation is correct, the cosmic radiation shows an intrinsic anisotropy of less than one part in $3000\left(1 \mathrm{~m}^{\circ} \mathrm{K}\right)$. The polarization properties of the radiation have been largely unexplored although in their original paper on the discovery of the background radiation, Penzias and Wilson assert that this radiation is "within the limits of our observations...unpolarized...". 6

It is of great interest to measure the polarization because of its potential to detect and distinguish deviations from the simple Big Bang model. Rees has shown that any intrinsic anisotropy in the cosmic radiation present at the time of decoupling or last scattering manifests itself as a linear polarization through the mechanism of Thomson scattering. ${ }^{7}$ Similarly, a last scattering by matter with a large velocity in the co-moving frame produces a net polarization. The predicted polarization anisotropy is highly model dependent, but is estimated to be on the order of one to one-hundredth of the intrinsic intensity anisotropy.

Recent reports of the first order anisotropy and the deviations from a blackbody spectrum provide added stimulus for investigating the polarization. We report here preliminary measurements made at $9 \mathrm{~mm}$ wavelength covering three circles at northern declinations $(\delta)$ of $38^{\circ}, 53^{\circ}$ and $63^{\circ}$.

The polarimeter is shown schematically in Figure 1 . The antenna is a dual-mode, corrugated-horn having nearly equal gain patterns for both linear polarizations. ${ }^{8}$ Below the antenna is a Faraday rotation switch which alternately rotates the incoming planes of polarization from $+45^{\circ}$ to $-45^{\circ}$ at $100 \mathrm{~Hz}$. Between the switch and the receiver is an element which selects one of the linear polarizations. Therefore, the input signal to the receiver switches rapidly between two orthogonal linear polarizations. The output of 
the receiver is analyzed for a signal synchronous with the switching of the Faraday rotator. Thus, the apparatus measures the difference in power between the two orthogonal polarization states. The information is digitized and recorded on magnetic tape. In addition, the polarimeter physically rotates in $45^{\circ}$ increments around the beam axis at 100 second intervals. This rotation allows us to measure both orthogonal components of any possible polarized signal and provides a means for distinguishing between external signals and imbalances in the apparatus.

The antenna ( $7^{\circ}$ beamwidth) has very low side lobes, such that the measured response in all directions more than $90^{\circ}$ from the antenna axis is reduced by a factor of $10^{8}$ over the forward gain. The addition of a ground shield further suppresses the effects of the earth, so that the total intensity received from the earth is less than $0.2 \mathrm{~m}^{\circ} \mathrm{K}$. We estimate the apparent polarized signal resulting from this ground pickup to be less than $0.05 \mathrm{~m}^{\circ} \mathrm{K}$. The Faraday rotation switch is a ferrite device and thus is sensitive to temperature and magnetic fields. The switch is temperature controlled to $0.2^{\circ} \mathrm{C}$ and shielded against external magnetic fields. The ferrite has a temperature dependent absorption coefficient of about $5 \mathrm{~m}^{\circ} \mathrm{K} /{ }^{\circ} \mathrm{C}$. Thermal mass insures that the ferrite temperature does not change faster than $0.3^{\circ} \mathrm{C}$ per hour. Hence even without the small correction we make in the data analysis, the ambient temperature changes could not contribute more than $0.08 \mathrm{~m}^{\circ} \mathrm{K}$ signal for our $100 \mathrm{sec}$ integration time. The ferrite also has a magnetization dependent absorption coefficient. Tests indicate that the effect of the earth's field is less than $0.08 \mathrm{~m}^{\circ} \mathrm{K}$ at the $95 \%$ confidence level. The receiver is a double-balanced mixer with a sensitivity of $100 \mathrm{~m}^{\circ} \mathrm{K} / \mathrm{Hz}{ }^{1 / 2}$, a center frequency of $33.1 \mathrm{GHz}$ and a bandwidth of $500 \mathrm{MHz}$. 
A polarized source calibrates the system. The source consists of a grid of copper strips on a thin plastic sheet and an ambient blackbody source. The calibrator produces a polarized signal whose amplitude $\left(280^{\circ} \mathrm{K}\right)$ is the difference between the ambient blackbody source $\left(295^{\circ} \mathrm{K}\right)$ and the sky $\left(15^{\circ} \mathrm{K}\right)$.

The measurements were made at the Lawrence Berkeley Laboratory $\left(38^{\circ} \mathrm{N}\right.$ latitude) during June, July, and August of 1978 . The data at $38^{\circ}$ declination were obtained by pointing the instrument vertically while the $53^{\circ}$ and $63^{\circ}$ declination data were taken by tilting north $15^{\circ}$ and $25^{\circ}$ respectively.

We analyzed the data by first calculating two Stokes parameters, $Q=T_{N S}-T_{E W}$ and $U=T_{N W, S E}-T_{N E}, S W^{*}$ Thus, for example, $Q$ is the difference in temperature of the North-South and East-West polarizations. $Q$ and $U$ are signal-averaged into hourly bins with a 24 siderial hour period. The largest source of extraneous polarized radiation is expected to be synchronous radiation from the galaxy. We have used data from polarization surveys at 820 $\mathrm{MHz}^{9}{ }^{9} 10$, combined with extrapolations of low frequency intensity measurements, to estimate the polarized signal at $33 \mathrm{GHz}$. The polarized component of the galactic synchrotron signal should be less than $0.05 \pm 0.04 \mathrm{~m}^{\circ} \mathrm{K}$ over all the areas observed. We therefore conclude that the signal-averaged data shown in Figure 2 are a good measure of any possible cosmic signal. The data are fit to a constant (D.C.) level and periods of 12 and 24 hours, excluding the sun-contaminated portion of the vertical $\left(\delta=38^{\circ}\right)$ data. We find no evidence for linear polarization in the cosmic background radiation and set the $95 \%$ confidence level limits shown in Table I. Table II summarizes the current status of polarization measurements.

Since all measurements are consistent with no or very little polarization, one must consider the possibility that depolarization mechanisms destroy intrinsic polarization. Our calculations indicate that Faraday rotation, 
the birefringence of a plasma in a magnetic field, is small for the galaxy and for the ionosphere. A universal magnetic field of magnitude greater than $10^{-7} \mathrm{~g}$ and an electron density of $10^{-5} \mathrm{~cm}^{-3}$ could effectively depolarize a signal, though there is no evidence that such fields exist. Measurements ${ }^{13}$ of the polarization of extragalactic objects, particularly quasars with redshifts $z \approx 2$ indicate that depolarization through Faraday rotation at $33 \mathrm{GHz}$ is not important to at least a redshift $z \geqslant 10$. Brans $^{14}$ has shown that the anisotropic expansion itself may tend to rotate the polarization, through the metric anisotropies, but this effect has been shown to be smal1. ${ }^{12,15}$

The null result observed is consistent with a thermal origin for the cosmic background radiation and an isotropic expansion. Using the calculations of Rees and assuming recombination at $z \sim 1500$ with no subsequent reheating, our limit of $0.8 \mathrm{~m}^{\circ} \mathrm{K}$ implies a present differential expansion limit of about one part in $10^{6}$. If the primordial plasma is re-ionized after recombination, the limit on the present differential expansion rate is about a part in $10^{4}$ for a critically dense universe. The intensity anisotropy is reduced by Thomson scattering while the polarization results from this scattering. The present intensity anisotropy roughly equals the Hubble expansion anisotropy shortly after the last scattering, while the polarization anisotropy remains at the value produced at the last few scatterings. The Hubble anisotropy decreases as the universe evolves. Thus, in the case of no reionization after recombination the $1 \mathrm{~m}^{\circ} \mathrm{K} 1$ imit ${ }^{5}$ on the intensity anisotropy implies a more stringent limit on the present differential expansion rate whereas in the case of reheating after recombination a comparable limit is obtained. The limits on the rms roughness of the sky imply that gravitational waves with wavelengths which are large (> few hundred $\mathrm{Mpc}$ ) on a cosmic scale have an energy density which is much lower than is needed to close the universe. ${ }^{12}$ 
This work was supported in part by the U.S. Department of Energy and the National Aeronautics and Space Administration. One of the us (P.L.) gratefully aknowledges support from the National Science Foundation as a NSF Fellow. We are indebted to $\mathrm{C}$. Witebsky for calculating the galactic backgrounds and to L.W. Alvarez, J.S. Aymong, A. Buffington, L. Deutch, L. Drexler, H.B. Dougherty, J.H. Gibson, M.V. Gorenstein, T.S. Mast, R.A. Muller, and H.J. Yamada for support and valuable suggestions. 


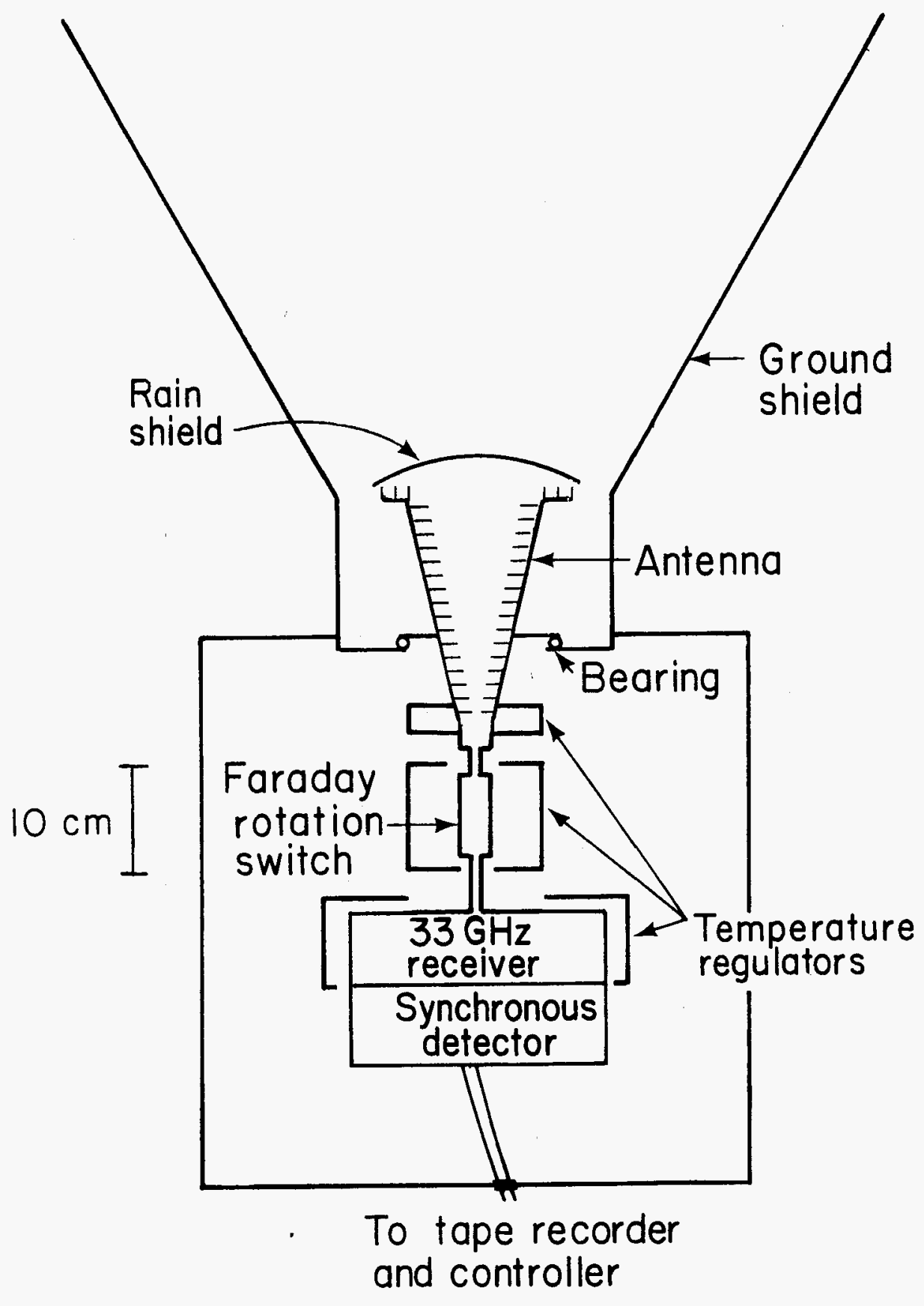

XBL $787-1395$

Fig. I Schematic of polarimeter 


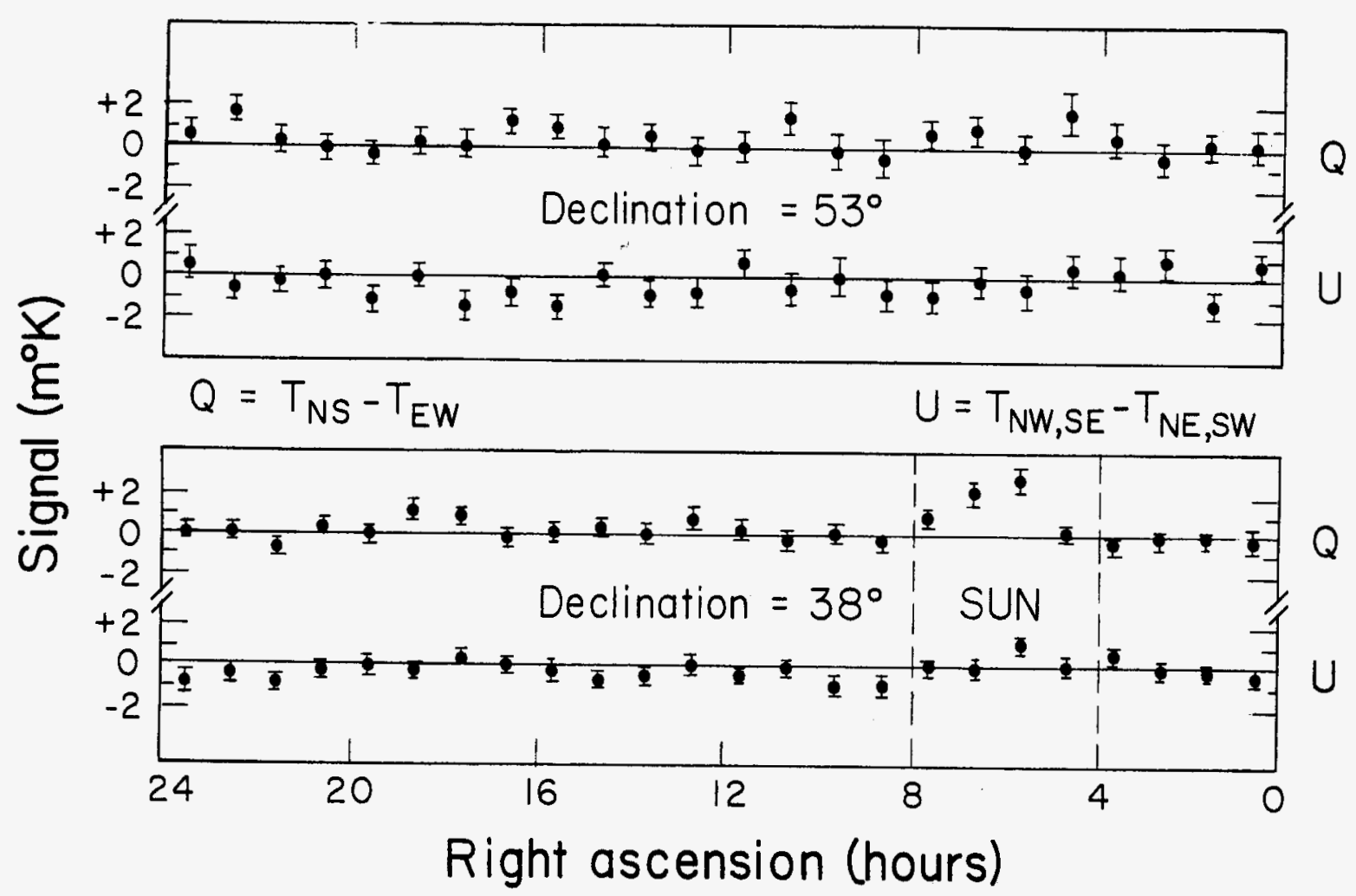

XBL $789-1544$

Fig. 2 Measured Stokes parameters $Q$ and $U$ as a function of position in the sky. Data eliminated because of sidelobe contamination by the sun is indicated. 
TABLE I

LIMITS ON POLARIZATION ANISOTROPIES 95\% CONFIDENCE LEVEL

(millidegrees Kelvin)

$\begin{array}{lcccccc}\text { Period } & \mathrm{Q} & \mathrm{U} & \mathrm{Q} & \mathrm{U} & \mathrm{Q} & \mathrm{U} \\ \text { DC } & 0.3 & 0.6 & 0.8 & 0.6 & 0.4 & 1.1 \\ 24 \text { hour } & 0.8 & 0.7 & 0.4 & 0.8 & 0.8 & 0.8 \\ 12 \text { hour } & 0.4 & 0.6 & 0.5 & 0.7 & 0.8 & 0.9 \\ \text { Sky Roughness } & & 0.6 & & 0.8 & & 0.7 \\ \text { Days of Data } & & & & & & \end{array}$

The limits are the magnitudes of the measured values plus two standard deviations. The sky roughness is the rms fluctuation on a $7^{\circ}$ scale size.

TABLE II

MEASURED LIMITS ON LINEAR POLARIZATION 95\% CONFIDENCE LEVEL

\begin{tabular}{|c|c|c|c|}
\hline Reference & Wavelength (CM) & Sky Coverage & Limit \\
\hline Penzias and Wilson ${ }^{6}$ & 7.35 & scattered & $\begin{array}{l}Q, U / T \\
10 \%\end{array}$ \\
\hline Nanos $^{11}$ & 3.2 & $\delta=+40^{\circ}$ & $0.05 \%$ \\
\hline Th1s experiment & 0.9 & $\begin{aligned} \delta= & +38^{\circ} \\
& +53^{\circ}\end{aligned}$ & $0.03 \%$ \\
\hline & & $\delta=+63^{\circ}$ & $0.04 \%$ \\
\hline Caderni et al. ${ }^{12}$ & $0.05-0.3$ & $\begin{array}{l}\text { near galactic } \\
\text { center }\end{array}$ & $0.1-1 \%$ \\
\hline
\end{tabular}




\section{REFERENCES}

1. R. Hanbury Brown, and R.Q. Twiss, Nature 178, 1447 (1956).

2. L. Danese and G. De Zotti, Nuovo Cimento $\underline{7}, 3,277$ (1977).

3. D. Woody and P. Richards, Phys. Rev. Letters, to be published.

4. B.E. Corey and D.T. Wilkinson, Bu11. Astron. Astrophys. Soc. 8 , 351 (1976).

5. G.F. Smoot, M.V. Gorenstein, and R.A. Muller, Phys. Rev. Letters 39, 14, 898 (1977).

6. A.A. Penzias and R.W. Wilson, Astrophys. J. 142, 419 (1965).

7. M.J. Rees, Astrophys. J. 153, LI (1968).

8. M. Janssen, et al. IEEE, Antennas and Propagation, to be published.

9. E.M. Berkhuijsen, Astron. Astrophys. Supp1. 5, 263 (1972).

10. E.M. Berkhuijsen, Astron. Astrophys. 14, 359 (1971).

11. G.P. Nanos, Ph.D. Thesis, Princeton University, 1974 (unpublished).

12. N. Caderni, R. Fabbi, B. Melchiorri, F. Melchiorri, and V. Natale, Phys. Rev. D. 17,1901 (1978).

13. F. Gardner, D. Morris and J. Whiteoak, Aust. J. Phys. 22, 79 (1969).

14. C. Brans, Astrophys. J. 197, 1 (1975).

15. A.M. Amile and R.A. Breuer, Astrophys. J. 217, 353 (1977). 